\title{
Modeling of austenite to ferrite transformation
}

\author{
MOHSEN KAZEMINEZHAD* \\ Department of Materials Science and Engineering, Sharif University of Technology, Azadi Avenue, Tehran, Iran
}

MS received 17 January 2011; revised 9 July 2011

\begin{abstract}
In this research, an algorithm based on the $Q$-state Potts model is presented for modeling the austenite to ferrite transformation. In the algorithm, it is possible to exactly track boundary migration of the phase formed during transformation. In the algorithm, effects of changes in chemical free energy, strain free energy and interfacial energies of austenite-austenite, ferrite-ferrite and austenite-ferrite during transformation are considered. From the algorithm, the kinetics of transformation and mean ferrite grain size for different cooling rates are calculated. It is found that there is a good agreement between the calculated and experimental results.
\end{abstract}

Keywords. Model; phase transformation; steel; austenite; ferrite; kinetics.

\section{Introduction}

In heat treatments of steels, the austenite to ferrite transformation is an important phenomenon which determines the final microstructure and mechanical properties of steels. Many analytical and numerical methods of simulation have been presented for modeling the austenite to ferrite transformation (Umemoto et al 1986, 1987; Lan et al 2004; Tong et al 2004; Thiessen et al 2006; Singh et al 2007). In analytical methods, the Johnson-Mehl relationship is considered and the fraction of transformed phase is predicted (Umemoto et al 1986, 1987). In these methods the effects of nucleation phenomenon cannot be considered for different nucleation sites, i.e. interior of grain, grain boundaries and triple junction of boundaries, simultaneously. On the other hand, different equations are achieved for different nucleation sites and thus it is difficult to make an exact analysis about the transformation. The other modeling works are mainly on numerically solving the diffusion equations, Cellular Automata and Monte Carlo model using hexagonal cell lattice (Lan et al 2004; Tong et al 2004; Thiessen et al 2006; Singh et al 2007). In two former methods, the effect of interfacial energy is neglected which has a great effect on the boundary migration of phases during the transformation. In the latter method, the algorithm for modeling the boundary migration of phases is based on the tracking of the hexagonal cells. Also, the interfacial energies of austenite-austenite, ferrite-ferrite and austenite-ferrite are not distinguished during the transformation. An algorithm can help to track the boundary migration of the phases in an exact manner during the transformation by distinguishing their interfacial energies. Therefore, in this study, every efforts are made on presenting an algorithm based on the $Q$-state Potts model to simulate the austenite

\footnotetext{
*Author for correspondence (mkazemi@ sharif.edu)
}

to ferrite transformation considering distinguished interfacial energies between the phases.

\section{Experimental procedure}

The samples of a low carbon steel with the chemical composition shown in table 1 were heated to austenitizing temperature of $950^{\circ} \mathrm{C}$ and held for $860 \mathrm{~s}$. Then the samples were cooled to room temperature at different cooling rates of $7^{\circ} \mathrm{C} / \mathrm{s}, 15^{\circ} \mathrm{C} / \mathrm{s}$ and $35^{\circ} \mathrm{C} / \mathrm{s}$. It should be mentioned that the cooling rates of the samples were measured using a $\mathrm{Ni}-\mathrm{NiCr}$ thermocouple. A thermometer was used to record the temperature data at time intervals of $1 \mathrm{~s}$. After metallographic examinations were carried out on the cooled specimens, it was found that the microstructures consisted of ferrite phase and negligible amount of pearlite (lower than 3\%). The ferrite grain size of each sample was measured according to the ASTM standard.

\section{Modeling austenite to ferrite transformation}

An algorithm based on the $Q$-state Potts model was developed to simulate the austenite to ferrite phase transformation. To do so, the initial austenite microstructure was generated on a two-dimensional triangular lattice using the normal grain growth algorithm of Monte Carlo model and considering the random integers from 1 to 64 as the orientations of grains (Anderson et al 1984). For modeling the

Table 1. Chemical composition of low carbon steel (wt\%).

\begin{tabular}{lllll}
\hline $\mathrm{C}$ & $\mathrm{Si}$ & $\mathrm{Mn}$ & $\mathrm{S}$ & $\mathrm{P}$ \\
\hline 0.07 & 0.02 & 0.45 & 0.012 & 0.017 \\
\hline
\end{tabular}


austenite to ferrite transformation, ferrite nucleation phenomenon should be modeled. As it has been reported in previous works (Umemoto et al 1987; Kazeminezhad and Karimi Taheri 2004), the nucleus density depends on the cooling rate from austenitizing temperature and the dependency is given as follows:

$$
n_{\alpha}=C_{1}(\dot{T})^{m},
$$

where $\dot{T}$ and $m$ are the cooling rate from austenitizing temperature and a positive constant, respectively. The value of $C_{1}$ sets the fraction of nucleus density on the lattice sites in the order of $10^{-2}$ (Srolovitz et al 1986).

Through austenite to ferrite transformation, the normal grain growth should be provided for both phases and also the phases should have different characteristics. Thus, the ferrite nucleus is determined by selecting one site randomly, and assigning a random integer from 65 to 128 . In other words, in order to make normal grain growth for ferrite as a new phase transformed from austenite, 64 states are considered as the orientations of ferrite grains. Also, integer range considered for ferrite phase is different from that considered for austenite phase which makes up different characteristics. Since the ferrite nucleation mainly occurs on the austenite grain boundaries (Umemoto et al 1987), those austenitic sites that have at least two unequal neighbours orientations are selected as the ferrite nuclei. The growth of ferrite nuclei is tracked by calculating the change in Hamiltonian energy due to reorientation attempts of the neighbours' sites of nuclei. In phase transformation of austenite to ferrite, free energy of the system is reduced. The contribution of different energies in changing free energy of the system during transformation are (Tong et al 2004; Thiessen et al 2006): (i) difference between chemical free energies of austenite and ferrite, (ii) a certain strain energy due to different lattice system of the phases, (iii) change in grain boundary energy of austenite due to nucleation of ferrite and thus austenite grain boundary removal, (iv) introducing the interfacial energy between austenite and ferrite during nucleation and ferrite grain growth and (v) conversion of the interface between ferrite and austenite to the interface of ferrite grains at the end of transformation.

On the basis of the above description the change in Hamiltonian energy can be expressed as follows:

$$
\begin{aligned}
\Delta H=( & \left.\Delta G_{\mu}+\Delta G_{\varepsilon}\right) f\left(S_{i}, S_{k}\right) \\
& +\sum_{j=1}^{6}\left[J_{\gamma-\gamma} g\left(S_{k}, S_{j}\right)+J_{\alpha-\gamma} h\left(S_{k}, S_{j}\right)\right. \\
& \left.+J_{\alpha-\alpha} p\left(S_{k}, S_{j}\right)\right]\left(1-\delta_{S_{k} S_{j}}\right) \\
& -\left[J_{\gamma-\gamma} g\left(S_{i}, S_{j}\right)+J_{\alpha-\gamma} h\left(S_{i}, S_{j}\right)\right. \\
& \left.+J_{\alpha-\alpha} p\left(S_{i}, S_{j}\right)\right]\left(1-\delta_{S_{i} S_{j}}\right),
\end{aligned}
$$

where $S_{i}$ is the original orientation of the randomly selected site, $S_{k}$ the newly assigned orientation to the selected site, $S_{j}$ the neighbours' orientations of the randomly selected site, $\delta_{i j}$ the Kronecker delta, $\Delta G_{\mu}$ the change in chemical free energy due to the reorientation of an austenite site to a ferrite site, $\Delta G_{\varepsilon}$ the strain energy due to the ferrite nucleation on the austenite site, $J_{\gamma-\gamma}$ the grain boundary energy between
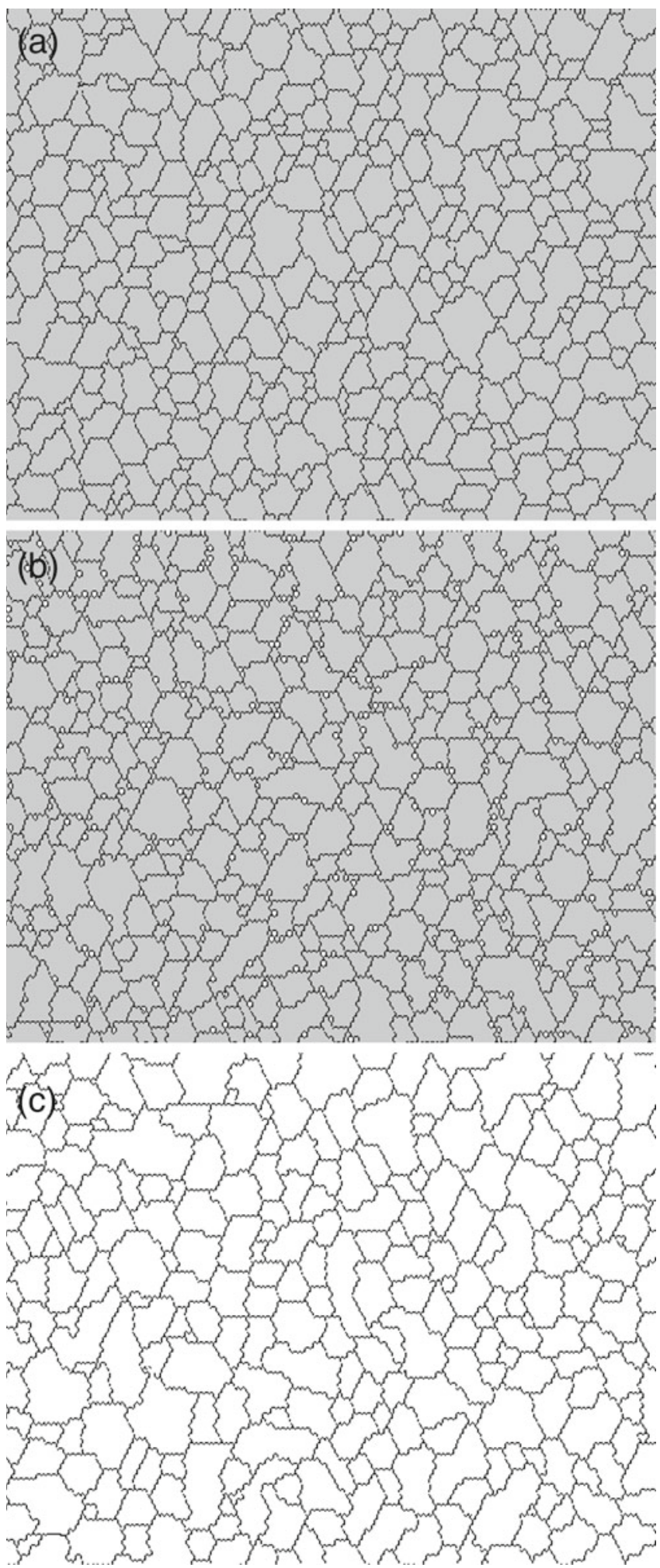

Figure 1. (a) Austenite microstructure, (b) ferrite nuclei on austenite microstructure and (c) ferrite microstructure. 
austenite grains, $J_{\alpha-\gamma}$ the grain boundary energy between austenite and ferrite grains, $J_{\alpha-\alpha}$ the grain boundary energy between ferrite grains. The functions $f, g, h$ and $p$ are defined as follows:

$$
\begin{aligned}
& f(x, y)= \begin{cases}1 & x \leq 64 \text { and } y>64 \\
0 & \text { in other cases }\end{cases} \\
& g(x, y)= \begin{cases}1 & x, y \leq 64 \\
0 & \text { in other cases }\end{cases} \\
& h(x, y)= \begin{cases}1 & (x>64, y \leq 64) \text { or }(x \leq 64, y>64) \\
0 & \text { in other cases }\end{cases} \\
& p(x, y)= \begin{cases}1 & x, y>64 \\
0 & \text { in other cases }\end{cases}
\end{aligned}
$$

In a specific reorientation attempt, if $\Delta H \leq 0$ then the reorientation is accepted. Otherwise, the reorientation should be stopped for the selected site.

It should be noted that the values of the parameters in modeling procedure can be found in an earlier study (Tong et al 2004).

\section{Results and discussion}

In figures $1(a)-(c)$, the achieved results from the modeling of austenite to ferrite transformation are exhibited. In figure 1(a), the austenite grains achieved from the normal grain growth algorithm of Monte Carlo model are observed. In the simulation procedure this microstructure is set for austenitizing heat treatment at $950^{\circ} \mathrm{C}$ and $860 \mathrm{~s}$. For a cooling condition with a rate of $15^{\circ} \mathrm{C} / \mathrm{s}$, the ferrite nuclei are observed as the white sites in figure 1(b) which are mainly generated on or near the austenite grain boundaries. According to the algorithm, the nuclei of ferrite grow and make the final microstructure of the steel (see figure 1c). In this regard,

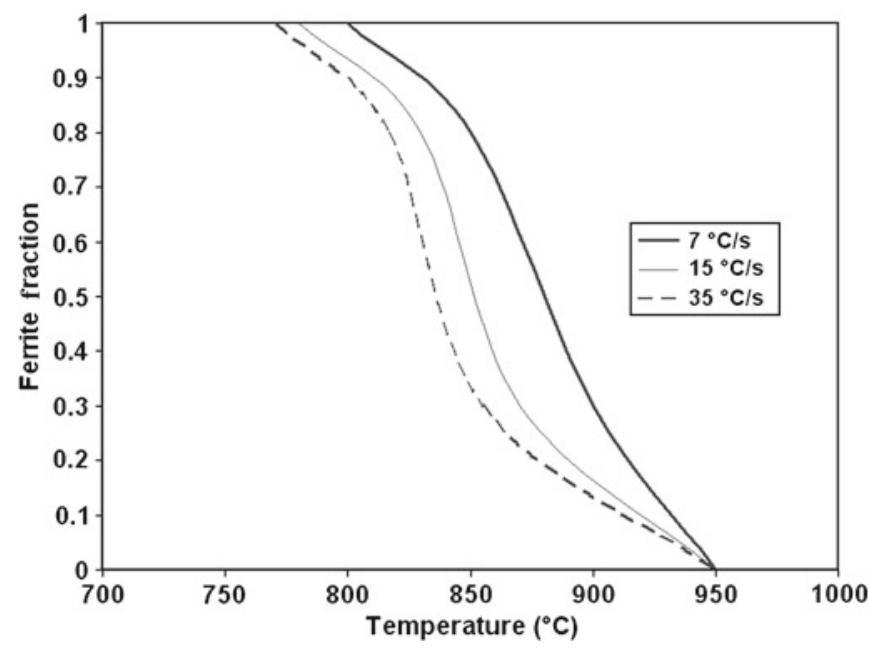

Figure 2. Simulated kinetics of austenite to ferrite transformation at different cooling rates.

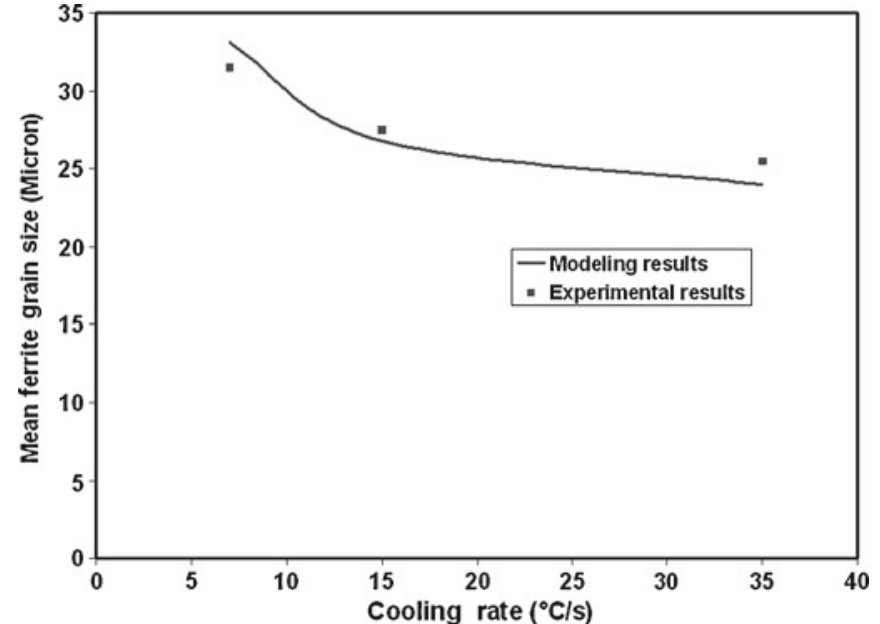

Figure 3. Comparison of simulated mean ferrite grain size with experimental one at different cooling rates.

the cooling rate from austenitizing temperature determines the nucleus density of ferrite (see (1)) and finally the grain size of transformed microstructure. Considering the reorientation process in the $Q$-state Potts algorithm based on the presented Hamiltonian energy, the fraction of ferrite formed during cooling of steel or kinetics of the transformation of austenite to ferrite is determined and presented for different cooling rates in figure 2 . As can be seen, with decreasing temperature (or cooling) the fraction of ferrite formed from the austenite phase is increased. Also, with increasing cooling rate a specific fraction of ferrite is formed in lower temperature. These trends agree well with the theoretical and experimental results reported by other researchers (Lan et al 2004; Tong et al 2004; Singh et al 2007). The mean grain sizes of ferrite were measured from the simulated microstructures for different cooling rates. In figure 3 , the mean ferrite grain sizes are plotted vs cooling rates. Also, in this figure the simulated grain sizes are compared with the experimental data. As observed, there is a good agreement between the simulated and experimental results. Moreover, figure 3 exhibits that with increasing cooling rate, the ferrite grain size is decreased. This is attributed to the increase of nucleus density of ferrite phase due to increase in cooling rate.

\section{Conclusions}

On the basis of the presented algorithm, the transformation of the austenite to ferrite is modelled. The kinetics of transformation and mean ferrite grain sizes formed at different cooling rates are predicted from the algorithm. It is found that there is a good agreement between predicted and experimental results. The predicted results show that at a specific temperature with increasing cooling rate, the fraction of ferrite formed during transformation is decreased. Also, the predicted mean ferrite grain size as well as experimental one are decreased with increasing cooling rate. 


\section{Acknowledgements}

The author wishes to thank the Research Board of Sharif University of Technology for providing research facilities.

\section{References}

Anderson M P, Srolovitz D J, Grest G S and Sahni P S 1984 Acta Metall. 32783

Kazeminezhad M and Karimi Taheri A 2004 Mater. Sci. Technol. 20106
Lan Y J, Li D Z and Li Y Y 2004 Acta Mater. 521721

Singh S B, Krishnan K and Sahay S S 2007 Mater. Sci. Eng. $\mathbf{A 4 4 5}$ 310

Srolovitz D J, Grest G S and Anderson M P 1986 Acta Metall. 34 1833

Thiessen R G, Richardson I M and Sietsma J 2006 Mater. Sci. Eng. A427 223

Tong M, Li D Z and Li Y Y 2004 Acta Mater. 521155

Umemoto M, Ohtsuka H and Tamura I 1986 Acta Metall. 34 1377

Umemoto M, Guo Z H and Tamura I 1987 Mater. Sci. Technol. 3294 